\title{
Fatores associados ao atraso no tratamento cirúrgico primário de fissuras labiopalatinas no Brasil: uma análise multinível
}

\author{
Factors associated with the delay in primary surgical treatment \\ of cleft lip and palate in Brazil: a multilevel analysis
}

Giselle Firmino Torres de Sousa (https://orcid.org/0000-0002-5940-2595) ${ }^{1}$

Angelo Giuseppe Roncalli (https://orcid.org/0000-0001-5311-697X) ${ }^{1}$

${ }^{1}$ Programa de Pós-

Graduação em Saúde

Coletiva, Universidade Federal do Rio Grande do Norte. Av. Sen. Salgado Filho 1787, Lagoa Nova. 59056-000. Natal RN Brasil. giselleftorres@gmail.com

\begin{abstract}
This study analyzed the delay in the primary surgical treatment of patients with cleft lip and palate in Brazil performed under the Unified Health System (SUS), and the effect of the sociodemographic context and municipal socioeconomic indicators on access to this treatment (2009-2013). Data from the Decentralized Hospital Information System relating to all hospital authorization forms paid for primary cleft lip and/or palate surgeries and socioeconomic data (2010) was used. The ages recommended by the American Cleft Palate-Craniofacial Association (12 months for lip surgeries and 18 months for palate surgeries) were used as parameters. The prevalence of delay observed in primary lip surgeries was $66.4 \%$ and in palate surgeries was $71.2 \%$. The North and Northeast regions had the worst percentages of delay. Non-whites had a greater prevalence of delay in cleft lip surgeries with $P R=$ 1.40 (1.30-1.50) and cleft palate surgeries with $P R$ $=1.27$ (1.21-1.33). The multilevel analysis identified the influence of self-reported skin color and Human Development Index (HDI) on the delay of primary lip surgery, and of HDI on the delay of palate surgery. The importance of social determination on the access to primary cleft lip and palate surgeries was evident.

Key words Cleft lip, Cleft palate, Surgery, Epidemiologic factors, Health services
\end{abstract}

Resumo Investigou-se o atraso no tratamento cirúrgico primário de fissuras labiopalatinas no âmbito do Sistema Único de Saúde (SUS) do Brasil e o efeito do contexto sociodemográfico e de indicadores socioeconômicos municipais no acesso a esse tratamento (2009-2013). Utilizaram-se informações das autorizações de internação hospitalar pagas para cirurgias primárias de lábio e de palato e variáveis socioeconômicas municipais (2010). Aplicaram-se os parâmetros adotados pela American Cleft Palate-Craniofacial Association: cirurgia de fissura de lábio (FL) aos 12 meses e de fissura de palato (FP) aos 18 meses. Observouse uma prevalência de $66,4 \%$ de atraso para cirurgias de lábio e 71,2\% para cirurgias de palato, com piores percentuais nas regiões Norte $(83,8 \%$ para FL e $86,6 \%$ para FP) e Nordeste (69\% para FL e $75,2 \%$ para FP). Não brancos apresentaram maior prevalência de atraso nas cirurgias de fissuras de lábio, com $R P=1,40$ (IC 95\%: 1,30-1,50), $e$ de fissuras palatinas, com $R P=1,27$ (IC 95\%: 1,21-1,33). A análise multinivel identificou influência da cor autorreferida e do Índice de Desenvolvimento Humano Municipal (IDH-M) nos atrasos da queiloplastia primária e do IDH-M nos atrasos da cirurgia de palato. Ficou evidente a determinação social o o efeito de contexto no acesso a essas cirurgias no Brasil.

Palavras-chave Fissura labial, Fissura palatina, Cirurgia, Fatores epidemiológicos, Serviços de saúde 


\section{Introdução}

As fissuras labiopalatinas são as malformações congênitas mais comuns na região craniofacial. Sua prevalência média mundial é de 9,92 por 10.000 nascidos vivos ${ }^{1}$. No Brasil, essa taxa é estimada em 5,86 por 10.000 nascidos; ou seja, a cada 1.893 nascimentos, uma criança apresenta algum tipo de fissura labiopalatina ${ }^{2}$.

Além da importância epidemiológica, essas malformações têm impacto social, psicológico e econômico, uma vez que os custos provenientes das fissuras labiopalatinas em termos de morbidade, cuidados de saúde, exclusão social e desemprego resultam em um grande fardo para a saúde, a qualidade de vida e o bem-estar socioeconômico dos indivíduos afetados, de suas famílias e da sociedade ${ }^{3,4}$.

As alterações anatômicas, caracterizadas pela descontinuidade das estruturas de lábio, palato ou ambos podem causar problemas estéticos, funcionais e psicossociais ${ }^{5}$. As alterações funcionais incluem: dificuldades de alimentação e deglutição, efeitos sobre a fala, problemas de audição, restrição ao crescimento e desenvolvimento craniofacial, alterações dentais e infecções de ouvido e vias aéreas ${ }^{6-9}$. No que concerne às implicações psicossociais, a literatura menciona problemas de comportamento, insatisfação com a aparência facial, depressão, ansiedade, baixa autoestima e dificuldades de aprendizagem e de relacionamento interpessoal ${ }^{10}$.

Trata-se, portanto, de uma condição complexa que, na maioria dos casos, requer um tratamento longo, multiprofissional, especializado e múltiplas cirurgias ${ }^{4,5}$. Não existe consenso na literatura quanto ao melhor protocolo a ser seguido. O protocolo cirúrgico vai depender do tipo de fissura e do estado de saúde da criança. A cirurgia reparadora primária é a primeira etapa da abordagem terapêutica do portador de fissura labiopalatina e pode reduzir fortemente as sequelas estéticas e funcionais ${ }^{5,11}$. Em contrapartida, a realização dessa cirurgia após o período recomendado pode interferir no desenvolvimento normal da fala, na aceitabilidade social e no desempenho escolar das crianças afetadas ${ }^{12}$.

A época de realização do tratamento cirúrgico primário é tão importante para a reabilitação dos portadores de fissuras labiopalatinas que a distribuição etária de realização da primeira cirurgia é considerada um indicador simples de acesso. Nos países mais pobres, considera-se a proporção de crianças que tiveram suas fissuras reparadas nas faixas etárias de 1 a 4, 5 a 9, 10 a 14 e mais de 15 anos. Nos países ricos, onde o tratamento ocorre mais precocemente e os registros são melhores, diferenças de idade na reparação cirúrgica primária podem evidenciar desigualdades entre grupos raciais, étnicos ou de diferentes condições socioeconômicas ${ }^{13}$.

Problemas com a organização dos serviços, a desigualdade no acesso aos cuidados e a incerteza na obtenção do tratamento são generalizados em todo o mundo. A falta de recursos coloca o tratamento cirúrgico primário fora do alcance de milhares de crianças residentes em países em desenvolvimento. Esse fato pode aumentar as taxas de mortalidade neonatal nessas nações, devido a complicações relacionadas à desnutrição e a infecções nas crianças portadoras de fissuras labiopalatinas mais graves ${ }^{13,14}$.

No Brasil, desde 1993, a atenção à saúde de portadores de fissuras labiopalatinas é financiada pelo governo por meio do Sistema Único de Saúde (SUS). Em 1998, o Ministério da Saúde criou a Rede de Referência no Tratamento de Deformidades Craniofaciais (RRTDCF), com o objetivo reduzir a iniquidade no acesso e ordenar a oferta de serviços no SUS ${ }^{15}$. Atualmente, essa rede é formada por 28 hospitais credenciados para a realização de procedimentos integrados de reabilitação estético-funcional de portadores de fissuras labiopalatinas ${ }^{16}$. No entanto, apesar do grande número de unidades que realizam esse atendimento no Brasil, disparidades regionais e problemas de ordenação e hierarquização estão presentes, fazendo com que muitos pacientes sejam tratados tardiamente ou permaneçam sem tratamento ${ }^{17,18}$.

Os principais centros de tratamento de anomalias craniofaciais brasileiros seguem os padrões estabelecidos pela American Cleft $\mathrm{Pa}$ late-Craniofacial Association (ACPA) ${ }^{19}$, que recomenda o fechamento cirúrgico primário do lábio dentro dos primeiros 12 meses de vida, ou o mais precocemente possível desde que seja considerado seguro para a criança, e o fechamento cirúrgico do palato até a criança completar 18 meses de $\operatorname{idade}^{20}$. O tratamento adequado e no tempo oportuno, segundo as diretrizes estabelecidas pela ACPA, e o acesso ao cuidado cirúrgico das fissuras labiopalatinas foram recentemente colocados entre as principais prioridades na agenda de pesquisa pelo Centers for Disease Control and Prevention (CDC) nos Estados Unidos ${ }^{21}$.

Apesar do reconhecimento pela literatura científica dos benefícios que a realização dos cuidados cirúrgicos primários das fissuras labiopalatinas no período adequado pode trazer, estudos nacionais relacionados a essa temática 


\section{Método}

Este estudo foi realizado a partir da análise das autorizações de internação hospitalar (AIH) pagas pelo SUS em todo o país para procedimentos de cirurgias primárias de correção de fissuras labiopalatinas do período de 2009 a 2013. O atraso para a realização de cirurgias primárias de fissuras de lábio e de palato foi avaliado utilizando como parâmetro as idades recomendadas pela ACPA. Desse modo, considerou-se que houve atraso nas cirurgias de lábio realizadas após os 12 meses de idade e nas cirurgias de palato realizadas após os 18 meses.

Os arquivos com as informações reduzidas de todos os procedimentos hospitalares realizados no período de 2009 a 2013 foram obtidos no Sistema de Informação Hospitalar Descentralizado (SIHD) do site do Departamento de Informática do SUS (DATASUS), gerando um total de 57.840.587 registros.

Após a coleta dos dados, foram excluídos os campos que não tinham interesse para o estudo e selecionados apenas os registros onde o campo de diagnóstico principal que gerou a internação correspondia aos códigos CID-10, de fissuras labiopalatinas (Q35 - fenda palatina, Q36 - fenda labial e Q37 - fenda labial com fenda palatina), gerando um arquivo com 40.259 registros. Esses registros apresentavam, além do campo com o diagnóstico principal, outras informações, como o código do procedimento realizado, a data de nascimento, a idade, o sexo e a raça do paciente, o município de residência do paciente, $\mathrm{o}$ ano de competência, a data de internação, a unidade federativa e o município onde o procedimento foi executado.

As cirurgias primárias de lábio (queiloplastias) e de palato (palatoplastias) foram identificadas pelos códigos do procedimento, de acordo com a tabela unificada do sistema de gerenciamento da tabela de procedimentos, medicamentos e OPM (órteses, próteses e materiais especiais) do SUS. Os dados das AIH pagas para procedi-

mentos de cirurgias primárias de lábio e palato foram então organizados em um banco de dados para a análise estatística. Para permitir um melhor diagnóstico do atraso no tratamento cirúrgico primário das fissuras labiopalatinas, a idade do paciente foi calculada em meses, utilizando a diferença entre a data de nascimento e a data de internação. Esse banco de dados foi integrado a outro que apresentava dados socioeconômicos provenientes do Atlas de Desenvolvimento $\mathrm{Hu}-$ mano dos municípios brasileiros do ano de 2010 por meio de um linkage determinístico. Foram incluídos todos os municípios brasileiros que apresentaram pelo menos uma AIH autorizada para cirurgia primária de fissura labiopalatina no SUS, considerando o município de residência do paciente.

As variáveis independentes incluídas na análise do atraso no tratamento cirúrgico primário das fissuras labiopalatinas foram as sociodemográficas (sexo e raça) e as de contexto municipal (Índice de Desenvolvimento Humano Municipal - IDHM, taxa de analfabetismo aos 15 anos ou mais, índice de Gini, proporção de pobres, taxa de desocupação aos 18 anos ou mais e renda per capita). A utilização dessas variáveis socioeconômicas permitiu a análise do atraso do tratamento em diferentes conjunturas de desenvolvimento humano municipal, bem como em relação a qualidade de vida, escolaridade, desigualdade social, pobreza, ocupação e renda.

Também foi realizada a análise de dados perdidos e atípicos. No primeiro caso, foram identificadas menos de $10 \%$ de perdas e, constatada sua aleatoriedade, foram consideradas ignoráveis. Para a identificação dos dados atípicos, foi utilizada a medida $\mathrm{D}^{2}$ de Mahalanobis, que identifica dados atípicos multivariados.

Os dados relacionados ao atraso foram analisados por meio da estatística descritiva, por meio de frequências absolutas e relativas. Foi realizada a análise bivariada e o cálculo das RP (razões de prevalência) não ajustadas, seguidos pela análise de regressão multinível de Poisson, com o uso do software Stata 14. A modelagem multinível se iniciou com o modelo nulo (vazio), em que o efeito de contexto foi testado a partir das variáveis socioeconômicas em nível municipal. Esse modelo não se mostrou adequado, mesmo após criteriosa análise de dados perdidos e de valores atípicos. Os dados municipais foram então agregados em uma divisão regional proposta pelo Instituto Brasileiro de Geografia e Estatística (IBGE): as Regiões Intermediárias de Articulação Urbana (RIAU). Essa divisão faz um recorte do territó- 
rio brasileiro em 161 regiões, tendo como base critérios socioeconômicos e a concentração de atividades econômicas públicas e privadas. Nesse caso, no modelo final da análise foram identificados 4.999 casos para fissura labial e 10.107 para fissura palatina (nível individual) aninhados em 157 regiões (nível de contexto), uma vez que nem todas as 161 apresentaram desfecho ou continham valores perdidos para alguma variável. $\mathrm{O}$ modelo nulo com esse nível de agregação mostrou maior significância, sendo portanto utilizado como nível de contexto.

Uma vez observado o efeito significativo no modelo nulo, foram feitas as inclusões das variáveis de contexto e individuais. Foi realizada ainda a análise de interação cross-level, a partir da inclusão de um termo de interação entre variáveis de primeiro e segundo níveis. Para todas as análises, adotou-se o limite de significância de 5\%.

\section{Resultados}

O total de procedimentos cirúrgicos autorizados para o tratamento de fissuras labiopalatinas no Sistema Único de Saúde, no período de 2009 a 2013, foi de 40.259 procedimentos. O atraso no tratamento cirúrgico primário das fissuras labiopalatinas foi avaliado a partir da análise da idade de realização das cirurgias reparadoras primárias, totalizando 7.902 queiloplastias e 15.216 palatoplastias autorizadas pelo SUS no período do estudo.

A média de atraso do tratamento no quinquênio estudado foi de $66,4 \%$ para as cirurgias primárias de fissuras labiais e de $71,2 \%$ para as cirurgias primárias de fissuras palatinas.

O estudo identificou maior atraso para o tratamento cirúrgico primário das fissuras palatinas do que para o tratamento das fissuras labiais em todas as cinco regiões naturais. As regiões Norte e Nordeste apresentaram os piores resultados em relação ao atraso no tratamento dos dois tipos de fissuras labiopalatinas analisados, enquanto a região Sudeste apresentou os menores percentuais de retardo, com melhores resultados para as cirurgias labiais do que para as palatinas. A prevalência de atraso nas cirurgias de fissuras labiopalatinas entre 2009 e 2013, de acordo com a região natural de residência do paciente e o tipo de fissura, está ilustrada na Gráfico 1.

A análise bivariada das variáveis sociodemográficas mostrou significância estatística na associação da variável sexo com o atraso na realização da cirurgia primária de lábio e da vari- ável raça $(\mathrm{p}<0,001)$ com o atraso na realização de ambas as cirurgias (queiloplastia e palatoplastia). O atraso no tratamento cirúrgico primário da fissura de lábio mostrou-se sete pontos percentuais mais prevalente no sexo feminino. Em relação à variável raça, os resultados mostraram que o atraso no tratamento cirúrgico primário das fissuras palatinas é $27 \%$ mais prevalente em pacientes não brancos em comparação a pacientes brancos, e para as cirurgias primárias de lábio esse atraso em não brancos é ainda maior, chegando a $40 \%$.

Todas as variáveis socioeconômicas municipais incluídas no estudo mostraram significância estatística em relação ao atraso no tratamento cirúrgico primário das fissuras de lábio e de palato na análise bivariada e foram posteriormente incluídas na análise multinível. Para ambos os tipos de fissuras, as razões de prevalência de atraso no tratamento cirúrgico primário se mostraram maiores em pacientes residentes em regiões cujos municípios possuem menor IDH, maior taxa de analfabetismo aos 15 anos de idade, maior índice de Gini, maior percentual de pobreza e desocupação aos 18 anos ou mais, além de rendas per capita mais baixas. As frequências absolutas e relativas e razões de prevalência (IC 95\%) de atraso no tratamento cirúrgico primário de fissuras labiais e palatinas no SUS no período de 2009 a 2013 estão descritas na Tabela 1.

Nas duas modelagens de regressão multinível de Poisson, as variáveis relativas às taxas de analfabetismo, pobreza, desemprego e renda foram excluídas por colinearidade ou perderam a significância. Em ambos os casos, o modelo 1 é representado pelas variáveis de contexto, e o modelo 2 pelas variáveis individuais. No modelo para fissura labial (Tabela 2), permaneceram significativas a raça, para o nível individual, e o IDH, para o contexto. Foi realizada, ainda, análise de interação cross-level a partir da inclusão do termo de interação "IDH $\mathrm{x}$ raça", o qual não alterou significativamente os valores de variância e das estimativas, indicando ausência desse efeito. Para a fissura palatina ( $\mathrm{Ta}$ bela 3), a variável de contexto IDH e a variável raça permaneceram significativas, porém não ocorreu o ajuste do modelo 2, conforme se observa pelo teste likelihood ratio $(\mathrm{p}=1,000)$.

\section{Discussão}

Os resultados da análise multinível mostraram a importância da determinação social no atraso do tratamento cirúrgico primário das fissuras la- 


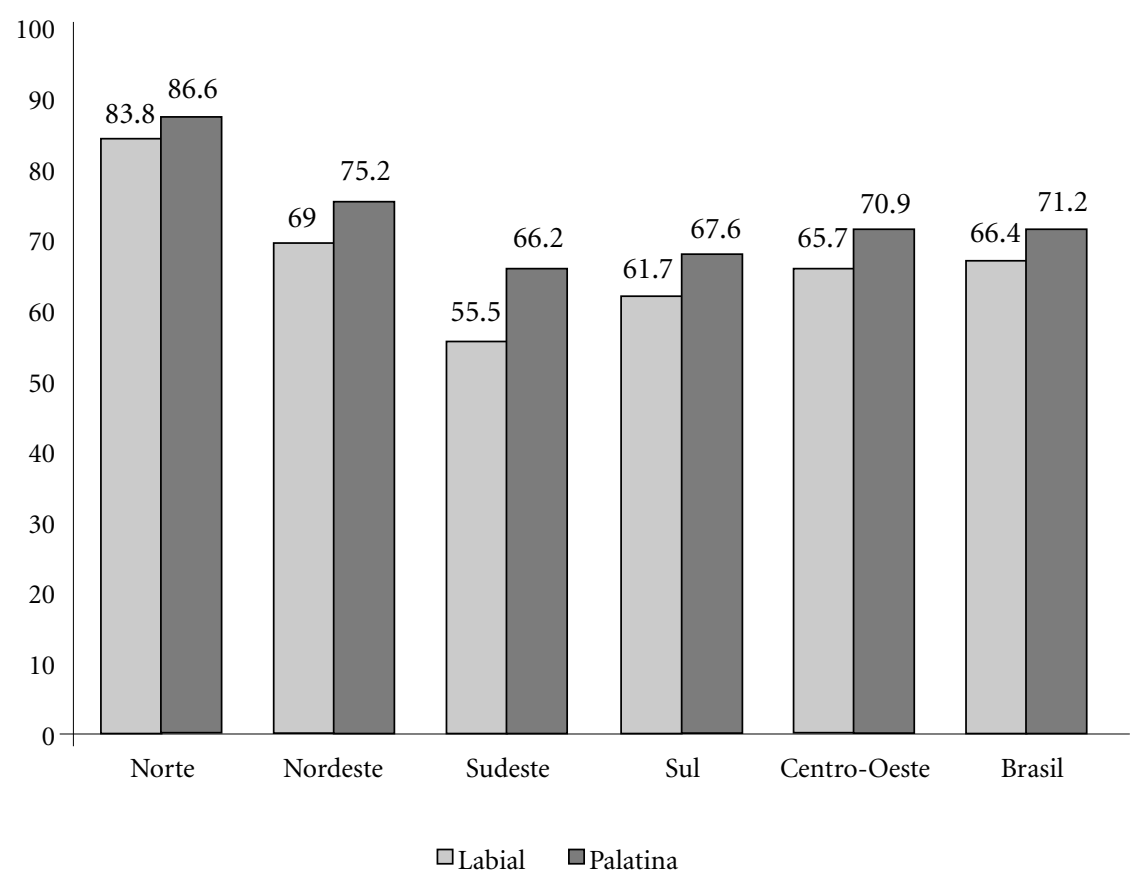

Gráfico 1. Prevalência de atraso na cirurgia primária de fissura labiopalatina entre 2009 e 2013, de acordo com a região e tipo de fissura. Brasil, 2017.

Fonte: SIHD/DATASUS.

biopalatinas em nosso país. As variáveis raça (no nível individual) e IDH-M (no nível do contexto) influenciam no atraso da realização da cirurgia primária de lábio. O IDH-M tem influência no atraso da realização da cirurgia primária de palato.

Os resultados reforçam ainda um forte efeito da variável raça sobre o atraso no tratamento cirúrgico das fissuras labiopalatinas no período avaliado. A desigualdade étnico-racial constitui um importante eixo no estudo das desigualdades em saúde. Embora a classificação por grupo racial não seja útil enquanto categoria biológica, é um importante constructo social que determina identidades, acesso a recursos e valorização na sociedade. No Brasil, tal desigualdade decorre principalmente de um contexto histórico de escravidão de afrodescendentes, que deixou marcas na posição social de sucessivas gerações da população negra ${ }^{24}$.

O campo cor/raça faz parte das informações registradas pelos sistemas brasileiros de informação em saúde desde 1995/1996. As categorias de classificação de raça seguem aquelas adotadas pelo IBGE (Portaria no $3.947 / G M)^{24}$. Apesar do alto número de informações perdidas no registro da variável raça observado neste estudo (36,4\% para procedimentos de queiloplastia e $29,5 \%$ para procedimentos de palatoplastia), a grande diferença em relação à prevalência observada no atraso da cirurgia primária das fissuras labiopalatinas entre os pacientes não brancos e brancos deixa evidente a desigualdade étnico-racial no acesso ao tratamento, resultante de diferenças socioeconômicas neste grupo.

A desigualdade entre grupos raciais em relação ao atraso no tratamento cirúrgico primário das fissuras labiopalatinas também foi observada nos Estados Unidos, em estudo realizado por Cassel e colaboradores ${ }^{25}$. No referido estudo, crianças de mães negras e de mães hispânicas tiveram, respectivamente, $70 \%$ e $14 \%$ menos chance de realizar a cirurgia primária até os 18 meses, quando comparadas a crianças com mães brancas.

Travassos e colaboradores ${ }^{26}$ mencionam que o acesso aos serviços de saúde no Brasil é fortemente influenciado pela condição social das 
Tabela 1. Frequência absoluta e relativa, significância estatística e razão de prevalência (IC 95\%) do atraso no tratamento cirúrgico primário de fissuras labiopalatinas no SUS de 2009 a 2013. Brasil, 2017

\begin{tabular}{|c|c|c|c|c|c|c|c|c|}
\hline \multirow[t]{2}{*}{ Variável } & \multicolumn{4}{|c|}{$\begin{array}{l}\text { Atraso no tratamento cirúrgico de fenda } \\
\text { labial }\end{array}$} & \multicolumn{4}{|c|}{$\begin{array}{l}\text { Atraso no tratamento cirúrgico de } \\
\text { fenda palatina }\end{array}$} \\
\hline & $\mathbf{n}$ & $\%$ & $\mathbf{p}$ & RP (IC 95\%) & $\mathbf{n}$ & $\%$ & $\mathbf{p}$ & RP (IC 95\%) \\
\hline \multicolumn{9}{|l|}{ Sexo } \\
\hline Masculino & 2.933 & 64,6 & & 1 & 4.372 & 70,6 & & 1 \\
\hline Feminino & 2.261 & 69,1 & 0,015 & $1,07(1,01-1,13)$ & 3.858 & 72,0 & 0,384 & $1,02(0,98-1,06)$ \\
\hline \multicolumn{9}{|l|}{ Raça } \\
\hline Brancos & 1.246 & 54,1 & & 1 & 3.048 & 65,2 & & 1 \\
\hline Não-brancos & 2.045 & 75,8 & $<0,001$ & $1,40(1.30-1,50)$ & 2.885 & 82,8 & $<0,001$ & $1,27(1,21-1,33)$ \\
\hline \multicolumn{9}{|c|}{ Índice de Desenvolvimento Humano Municipal (IDH-M) } \\
\hline 0,731 ou mais & 1.886 & 58,3 & & 1 & 3.473 & 66,2 & & 1 \\
\hline 0,647 a 0,731 & 1.278 & 64,1 & 0,002 & $1,12(1,04-1,20)$ & 2.456 & 71,1 & 0,006 & $1,07(1,02-1,13)$ \\
\hline Até 0,646 & 2.030 & 77,7 & $<0,001$ & $1,33(1,25-1,42)$ & 2.301 & 80,6 & $<0,001$ & $1,22(1,15-1,28)$ \\
\hline \multicolumn{9}{|c|}{ Taxa de analfabetismo da população de 15 anos ou mais de idade } \\
\hline Até 7,57 & 1.642 & 57,5 & & 1 & 3.587 & 66,3 & & 1 \\
\hline 7,58 a 16,86 & 2.274 & 71,5 & $<0,001$ & $1,24(1,17-1,32)$ & 2.845 & 74,6 & $<0,001$ & $1,13(1,07-1,18)$ \\
\hline 16,87 e mais & 1.278 & 72,0 & $<0,001$ & $1,14(1,16-1,35)$ & 1.798 & 77,1 & $<0,001$ & $1,16(1,10-1,23)$ \\
\hline \multicolumn{9}{|l|}{ Índice de Gini } \\
\hline Até 0,506 & 599 & 52,2 & & 1 & 1.778 & 64,7 & & 1 \\
\hline 0,507 a 0,544 & 1.394 & 63,6 & $<0,001$ & $1,11(1,08-1,34)$ & 2.625 & 70,1 & 0,008 & $1,08(1,02-1,15)$ \\
\hline 0,545 e mais & 3.201 & 71,6 & $<0,001$ & $1,21(1,26-1,50)$ & 3.827 & 75,6 & $<0,001$ & $1,17(1,10-1,23)$ \\
\hline \multicolumn{9}{|l|}{ Proporção de pobres } \\
\hline Até 7,90 & 1.886 & 58,3 & & 1 & 3.571 & 65,5 & & 1 \\
\hline 7,91 a 30,90 & 2.055 & 70,4 & $<0,001$ & $1,21(1,13-1,28)$ & 2.916 & 75,9 & $<0,001$ & $1,16(1,10-1,22)$ \\
\hline 30,91 e mais & 1.253 & 75,6 & $<0,001$ & $1,30(1,21-1,39)$ & 1.743 & 77,2 & $<0,001$ & $1,18(1,11-1,25)$ \\
\hline \multicolumn{9}{|c|}{ Taxa de desocupação aos 18 anos de idade ou mais } \\
\hline Até 5,80 & 1.017 & 55,5 & & 1 & 1.937 & 63,8 & & 1 \\
\hline 5,81 a 7,50 & 656 & 62,1 & 0,025 & $1,11(1,01-1,23)$ & 2.065 & 72,2 & $<0,001$ & $1,13(1,06-1,20)$ \\
\hline 7,51 e mais & 3.521 & 71,5 & $<0,001$ & $1,29(1,20-1,38)$ & 4.228 & 74,7 & $<0,001$ & $1,17(1,11-1,23)$ \\
\hline \multicolumn{9}{|c|}{ Renda per capita média } \\
\hline $\mathrm{R} \$ 802$ e mais & 1.886 & 58,3 & & 1 & 3.473 & 66,2 & & 1 \\
\hline $\mathrm{R} \$ 438$ a $\mathrm{R} \$ 801$ & 1.898 & 70,3 & $<0,001$ & $1,20(1,13-1,28)$ & 2.813 & 74,0 & $<0,001$ & $1,12(1,06-1,17)$ \\
\hline Até R\$ 437 & 1.410 & 75,1 & $<0,001$ & $1,28(1,20-1,38)$ & 1.944 & 77,7 & $<0,001$ & $1,17(1,11-1,24)$ \\
\hline
\end{tabular}

Fonte: Elaborado pelos autores.

pessoas e pelo grau de desenvolvimento econômico do local onde residem. Segundo Araújo ${ }^{27}$, as desigualdades regionais são uma extensão geográfica dos desníveis sociais e deveria caber aos serviços públicos, particularmente aos de saúde, o papel de redistribuir, corrigir e atenuar essas desigualdades, fato que não ocorre no Brasil, um país onde a distribuição regional dos serviços e dos recursos humanos na área da saúde é terrivelmente desigual.

Neste estudo, as macrorregiões Norte e Nordeste, que concentram os municípios com um maior número de pobres e os piores indicadores de saúde 28,29 , foram as que apresentaram a pior situação em relação ao atraso na realização das cirurgias primárias de fissuras labiopalatinas.

A análise dos dados mostrou que quanto menor o Índice de Desenvolvimento Humano do município, maior a prevalência de atraso no tratamento cirúrgico primário das fissuras labiais e palatinas. Carlson e colaboradores ${ }^{30}$ investigaram a relação entre o acesso tardio à palatoplastia primária após os 24 meses e o PIB per capita em 11 países em desenvolvimento (Gana, Etiópia, República Democrática do Congo, Madagascar, China, Índia, Nicarágua, Bolívia, Paraguai, Peru e 
Tabela 2. Modelo de regressão multinível de Poisson para o atraso no tratamento cirúrgico primário de fissura labial no SUS de 2009 a 2013, de acordo com fatores individuais e contextuais. Brasil, 2017.

\begin{tabular}{|c|c|c|c|c|c|}
\hline \multirow{2}{*}{ Variáveis } & \multirow{2}{*}{$\begin{array}{c}\text { Modelo nulo } \\
(\mathrm{n}=7.810) \\
\end{array}$} & \multicolumn{2}{|c|}{ Modelo $1(n=4.999)$} & \multicolumn{2}{|c|}{ Modelo $2(n=4.999)$} \\
\hline & & RP (IC 95\%) & p & RP (IC 95\%) & $\mathrm{p}$ \\
\hline \multicolumn{6}{|l|}{ Nível de contexto } \\
\hline \multicolumn{6}{|c|}{ Índice de Desenvolvimento Humano (IDH) } \\
\hline 0,731 e mais & & 1 & & 1 & \\
\hline 0,647 a 0,730 & & $1,04(0,93-1,16)$ & 0,509 & $1,05(0,93-1,19)$ & 0,433 \\
\hline Até 0,646 & & $1,17(1,04-1,32)$ & 0,009 & $1,26(1,11-1,44)$ & $<0,001$ \\
\hline \multicolumn{6}{|l|}{ Índice de Gini } \\
\hline Até 0,506 & & 1 & & & \\
\hline 0,507 a 0,544 & & $1,08(0,95-1,24)$ & 0,248 & & \\
\hline 0,545 e mais & & $1,16(0,99-1,35)$ & 0,063 & & \\
\hline \multicolumn{6}{|c|}{ Taxa de desocupação aos 18 anos ou mais de idade } \\
\hline Até 5,80 & & 1 & & & \\
\hline De 5,81 a 7,50 & & $0,99(0,87-1,14)$ & 0,929 & & \\
\hline 7,51 e mais & & $1,08(0,93-1,25)$ & 0,296 & & \\
\hline \multicolumn{6}{|l|}{ Nível individual } \\
\hline \multicolumn{6}{|l|}{ Sexo } \\
\hline Masculino & & & & 1 & \\
\hline Feminino & & & & $1,06(0,99-1,14)$ & 0,094 \\
\hline \multicolumn{6}{|l|}{ Raça } \\
\hline Brancos & & & & 1 & \\
\hline Não-Brancos & & & & $1,21(1,10-1,33)$ & $<0,001$ \\
\hline \multicolumn{6}{|l|}{ Efeitos fixos } \\
\hline Coeficiente (IC 95\%) & $-0,46(-0,52 ;-0,41)$ & $-0,64(-0,74 ;-0,54)$ & & $-0,70(-0,79 ;-0,61)$ & \\
\hline \multicolumn{6}{|l|}{ Efeitos aleatórios } \\
\hline Variância (IC 95\%) & $0,031(0,02-0,05)$ & $0,009(0,003-0,030)$ & & $0,010(0,003-0,038)$ & \\
\hline Alteração variância (\%) & - & 71,0 & & 67,7 & \\
\hline LR test $\left(x^{2}, p\right.$-value $)$ & $76,62(<0,001)$ & $5,11(0,012)$ & & $5,00(0,013)$ & \\
\hline
\end{tabular}

$\mathrm{IC}=$ intervalo de confiança; $\mathrm{RP}=$ razão de prevalência; $\mathrm{LR}=$ likelihood ratio.

Fonte: Elaborado pelos autores.

México) e constataram uma forte correlação negativa entre o status de renda nacional e o acesso tardio à cirurgia primária de fissura palatina, indicando um alto grau de desigualdade no acesso à cirurgia, sobretudo nos países de média e baixa rendas.

A American Cleft-Palate Association recomenda que a gestão dos pacientes com qualquer tipo de anomalia craniofacial seja conduzida por uma equipe interdisciplinar de especialistas que acompanhe um número suficiente de pacientes a cada ano para manter experiência clínica no diagnóstico e no tratamento ${ }^{19}$. Segundo Silva ${ }^{31}$, redes regionalizadas e integradas de atenção à saúde oferecem uma condição estrutural mais adequada à efetivação da atenção integral e reduzem os custos dos serviços por proporcionarem sua utilização de forma mais racional. Todavia, a grande disparidade entre as regiões geográficas brasileiras em relação ao atraso no tratamento cirúrgico primário das fissuras labiopalatinas observada neste estudo evidencia a existência de problemas na ordenação da oferta de serviços de alta complexidade nessa área.

O estudo de Monlleó e Gil-da-Silva-Lopes ${ }^{17}$ revelou concentração de centros de tratamento integrantes da Rede de Referência no Tratamento de Deformidades Craniofaciais (RRTDCF) na região Sudeste e um número insuficiente dos mesmo nas regiões Norte, Nordeste e Centro-Oeste. Segundo os autores, esse fato pode alimentar um importante fluxo de pacientes que buscam atendimento em instituições distantes do seu local de residência. Tal situação parece não ter sido alterada nos últimos anos. Atualmente, dos 28 hospitais habilitados pelo SUS para a realização 
Tabela 3. Modelo de regressão multinível de Poisson para o atraso no tratamento cirúrgico primário de fissura palatina no SUS de 2009 a 2013, de acordo com fatores individuais e contextuais. Brasil, 2017.

\begin{tabular}{|c|c|c|c|c|c|}
\hline \multirow{2}{*}{ Variáveis } & \multirow{2}{*}{$\begin{array}{c}\text { Modelo nulo } \\
(\mathrm{n}=15.071) \\
\end{array}$} & \multicolumn{2}{|c|}{ Modelo $1(n=15.071)$} & \multicolumn{2}{|c|}{ Modelo $2(n=10.107)$} \\
\hline & & RP (IC 95\%) & $\mathbf{p}$ & RP (IC 95\%) & $\mathbf{p}$ \\
\hline \multicolumn{6}{|l|}{ Nível de contexto } \\
\hline \multicolumn{6}{|c|}{ Índice de Desenvolvimento Humano (IDH) } \\
\hline 0,731 e mais & & 1 & & 1 & \\
\hline 0,647 a 0,730 & & $1,04(0,97-1,13)$ & 0,271 & $1,06(0,98-1,14)$ & 0,102 \\
\hline Até 0,646 & & $1,17(1,07-1,28)$ & $<0,001$ & $1,17(1,09-1,27)$ & $<0,001$ \\
\hline \multicolumn{6}{|l|}{ Índice de Gini } \\
\hline Até 0,506 & & 1 & & & \\
\hline 0,507 a 0,544 & & $0,97(0,88-1,05)$ & 0,456 & & \\
\hline 0,545 e mais & & $1,01(0,91-1,12)$ & 0,809 & & \\
\hline \multicolumn{6}{|c|}{ Taxa de desocupação aos 18 anos ou mais de idade } \\
\hline Até 5,80 & & 1 & & & \\
\hline De 5,81 a 7,50 & & $1,08(0,98-1,18)$ & 0,100 & & \\
\hline 7,51 e mais & & $1,07(0,97-1,19)$ & 0,177 & & \\
\hline \multicolumn{6}{|l|}{ Nível individual } \\
\hline \multicolumn{6}{|l|}{ Raça } \\
\hline Brancos & & & & 1 & \\
\hline Não-Brancos & & & & $1,18(1,11-1,26)$ & $<0,001$ \\
\hline \multicolumn{6}{|l|}{ Efeitos fixos } \\
\hline Coeficiente (IC 95\%) & $-0,44(-0,47 ;-0,41)$ & $-0,54(-0,61 ;-0,48)$ & & $-0,55(-0,59 ;-0,51)$ & \\
\hline \multicolumn{6}{|l|}{ Efeitos aleatórios } \\
\hline Variância (IC 95\%) & $0,014(0,009-0,024)$ & $0,007(0,003-0,014)$ & & $0,002(0,000-0,020)$ & \\
\hline Alteração Variância (\%) & - & 50,0 & & 85,7 & \\
\hline LR test $\left(x^{2}, p\right.$-value $)$ & $98,12(<0,001)$ & $29,95(<0,001)$ & & $0,00(1,000)$ & \\
\hline
\end{tabular}

de procedimentos integrados de reabilitação estético-funcional de fissurados, 19 estão localizados nas regiões Sul e Sudeste, e apenas cinco no Norte e Nordeste ${ }^{16}$. Essa disparidade pode ajudar a explicar os maiores percentuais de retardo no tratamento cirúrgico primário das fissuras labiopalatinas nas regiões Norte e Nordeste observados neste estudo.

Quanto ao tipo de fissura labiopalatina, foi observado maior atraso para o tratamento cirúrgico primário das fissuras de palato. Tal fato pode ser explicado, em parte, pela maior frequência de anomalias adicionais e à associação com síndromes nas crianças com fissuras de palato, quando comparadas com aquelas que apresentam fissuras de lábio, com ou sem envolvimento do palato ${ }^{3,32}$.

Devemos também considerar o fato de que as cirurgias reparadoras de palato são procedimentos mais complexos, que requerem maior experiência cirúrgica e anestésica por parte da equipe hospitalar, e que podem desenvolver complica- ções operatórias graves. Tais dificuldades podem fazer com que muitos centros de tratamento optem por não operar as fissuras palatinas mais complexas, reduzindo ainda mais a disponibilidade desse serviço ${ }^{33}$.

Estudo realizado na Carolina do Norte (EUA) observou que $88 \%$ das crianças com fissuras de lábio tiveram suas fissuras reparadas dentro do prazo recomendado pela ACPA, enquanto esse percentual foi bem menor (58\%) entre aquelas que apresentavam fissura de palato. No estudo mencionado, as crianças com fissuras isoladas apresentaram probabilidade $15 \%$ maior de passar por cirurgia até os 18 meses em relação às crianças com múltiplas anomalias, independentemente do tipo de fissura ${ }^{25}$. No estudo conduzido por Abbott e colaboradores ${ }^{12}$, também nos Estados Unidos, pacientes com diagnóstico de fissura labial com fissura palatina realizaram o reparo cirúrgico mais tardiamente do que aqueles que apresentavam apenas fissura palatina, re- 
sultado, possivelmente, da maior complexidade desse tipo de malformação.

Este estudo apresenta como limitação o fato de não incluir a análise de outros fatores individuais relacionados à situação clínica do paciente, como o grau de severidade da fissura, a associação com outros defeitos congênitos ou síndromes, baixo peso ao nascer e prematuridade, que podem ter relação com a realização do tratamento cirúrgico mais tardio. Tais informações estão disponíveis no Sistema de Informação sobre Nascidos Vivos (SINASC), mas não no Sistema de Informações Hospitalares do SUS (SIH/SUS). A falta de integração entre os diversos sistemas de informação em saúde podem, como no caso deste estudo, limitar as análises. $\mathrm{O}$ uso da técnica do linkage probabilístico poderia ser uma opção para identificar eventos não disponíveis em uma das duas fontes ${ }^{34}$. No entanto, o uso dessa técnica requer a utilização de dados primários com identificação do nome, o que não foi possível para este estudo.

Outra limitação apresentada diz respeito à cobertura do SIH/SUS, cujas informações estão restritas às internações realizadas no âmbito do SUS (hospitais públicos e privados conveniados), excluindo as hospitalizações custeadas diretamente, aquelas cobertas por seguros privados de saúde ou realizadas por organizações não governamentais. Entretanto, é importante ressaltar que tal cobertura é estimada em torno de $80 \%$ do total de internações do país, apresentando variações regionais ${ }^{35}$.

A falta de delimitação da área de cobertura dos hospitais habilitados pelo SUS para a realização de procedimentos de reabilitação dos portadores de fissuras labiopalatinas e o fato de muitos pacientes procurarem os hospitais dos maiores centros para realização do tratamento cirúrgico inviabilizaram a utilização da variável "proximidade dos serviços de reabilitação” neste estudo.
Quanto aos pontos positivos deste trabalho, podemos destacar: o fato de ser o primeiro estudo sobre o atraso no tratamento cirúrgico primário de fissuras labiopalatinas de abrangência nacional; a inclusão de dados individuais, e não apenas agregados; e o uso do código do diagnóstico principal (de acordo com a CID-10) vinculado ao código do procedimento cirúrgico, aumentando a confiabilidade do dado.

Embora crianças não operadas possam sobreviver, elas poderão enfrentar, ao longo de suas vidas, além da deformidade facial, problemas ao se alimentar, falar e ouvir, ter baixa autoestima e depressão, sofrer estigmatização e exclusão social e encontrar obstáculos ao emprego ${ }^{13}$. Temos que considerar que quanto mais o tratamento cirúrgico primário for retardado, maiores serão os impactos dessas condições na saúde e na qualidade de vida dessas crianças e de seus familiares.

$O$ estudo identificou que o atraso no tratamento cirúrgico primário das fissuras labiopalatinas no âmbito do Sistema Único de Saúde é maior em crianças que vivem em um contexto socioeconômico desfavorável, evidenciando a determinação social no retardamento desse tratamento e deixando claro que o sistema de saúde não está sendo capaz de assegurar o uso equânime dos serviços de reabilitação oferecidos aos portadores dessas enfermidades no Brasil.

É incontestável que o Estado deve garantir, via SUS, a atenção à saúde nessa área. No entanto, para a garantia do acesso à saúde dos portadores de fissuras labiopalatinas no tempo oportuno no Brasil, aumentar a oferta de serviços pode não ser suficiente. É essencial que haja uma ampla articulação intersetorial, com a implantação de políticas públicas de combate à pobreza e à desigualdade que assegurem a igualdade no direito de acesso ao tratamento e promova a melhoria das condições de vida e de saúde desses pacientes e de suas famílias. 


\section{Colaboradores}

GFT Sousa foi responsável pela coleta de dados nos sistemas de informação em saúde, pela redação final e análise estatística; AG Roncalli foi responsável pela coleta de dados nos sistemas de informação em saúde, pela redação final e pela supervisão da análise estatística.

\section{Referências}

1. IPDTOC Working Group. Prevalence at birth of cleft lip with or without cleft palate.propor data from the International Perinatal Database of Typical Oral Clefts (IPDTOC). Cleft Palate Craniofac J 2011; 48(1):66-81.

2. Sousa GFT, Roncalli AG. Orofacial clefts in Brazil and surgical rehabilitation under the Brazilian National Health System. Braz Oral Res 2017; 31:e23.

3. Monlleó IL, Barros AGR, Fontes, MIB, Andrade AKM, Brito GM, Nascimento DLL, Gil-da-Silva-Lopes VL. Diagnostic Implications of asssociated defects im patientes with typical orofacial clefts. J Pediatr 2015; 91:485-492.

4. Word Health Organization (WHO). Global strategies to reduce the health-care burden of craniofacial anomalies. Geneva: WHO; 2002.

5. Freitas JAS, Neves LT, Almeida ALPF, Garib DG, Trindade-Suedam IK, Yaedu RYF, Lauris RCMC, Soares S, Oliveira TM, Pinto JHN. Rehabilitative treatment of cleft lip and palate: experience of the Hospital for Rehabilitation of Craniofacial Anomalies - USP (HRAC-USP) - Part 1: overall aspects. J Appl Oral Sci 2012; 20(1):9-15.

6. Amstalden-Mendes LG, Gil-Da-Silva-Lopes VL. Fenda de lábio e/ou palato: recursos para a alimentação antes da correção cirúrgica. Rev de Cien Med 2006; 15(5):437-448.

7. Freitas JAS, Garib DG, Oliveira T, Lauris RCMC, Almeida ALPF, Neves LT, Trindade-Suedam IK, Yaedú RYF, Soares S, Pinto JHN. Rehabilitative treatment of cleft lip and palate: experience of the Hospital for Rehabilitation of Craniofacial Anomalies - USP (HRAC-USP) - Part 2: Pediatric Dentistry and Orthodontics. J Appl Oral Sci 2012; 20(2):272-285.

8. Silva DP, Dornelles S PLEA. Aspectos patofisiológicos do esfíncter velofaríngeo nas fissuras palatinas. Arq Int Otorrinolaringol 2008; 12(3):426-435.

9. Tannure PN, Oliveira CAGR, Maia LC, Vieira AR, Granjeiro JM, Costa MC. Prevalence of dental anomalies in nonsyndromic individuals with cleft lip and palate: A systematic review and meta-analysis. Cleft Palate Craniofac J 2012; 49(2):194-200.

10. Hunt O, Burden D, Hepper P, Johnston C. The psychosocial effects of cleft lip and palate: a systematic review. Eur J Orthod 2005; 27(3):274-285.

11. Alonso N, Sakai DY, Tanikawa, Lima-Junior JE, Rocha DL, Sterman S, Ferreira MC. Fissuras labiopalatinas: protocolo de atendimento multidisciplinar e seguimento longitudinal em 91 pacientes consecutivos. Rev Bras Cir Plast 2009, 24(2):176-181.

12. Abbott MM, kokoroski PJ, Meara JG. Timeliness of surgical care in children with special health care needs: delayed palate repair for publicly insured and minority children with cleft palate. J Pediatric Surg 2011; 46(7):1319-1324.

13. Mossey PA, Shaw WC, Munger RG, Murray JC, Murthy J, Little J. Global oral health inequalities: challenges in the prevention and management of orofacial clefts and potential solutions. Adv Dent Res 2011; 23(2):247-258 
14. Cubitt J, Hodges A, Galiawango G, Lierde KMV. Malnutrition in cleft lip and palate children in Uganda. Eur J Plast Surg 2012; 35:273-276.

15. Monlleó IL. Anomalias craniofaciais, genética e saúde Pública: contribuições para o reconhecimento da situação atual da assistência no Sistema Único de Saúde [dissertação]. Campinas: Faculdade de Ciências Médicas; 2004.

16. Departamento de Informática do SUS. Sistemas e aplicativos. CNES - Cadastro Nacional de Estabelecimentos de Saúde. Indicadores Habilitações [Internet]. [acessado 2016 Jun 23]. Disponível em: http:// cnes2. datasus.gov.br/Mod_Ind_Habilitacoes_Listar.asp? VTipo $=0401 \&$ VListar $=1 \&$ VEstado $=00 \&$ VMun $=\& V-$ Comp $=\& V$ Contador $=28 \& V$ Titulo $=\mathrm{H}$.

17. Monlleó IL, Gil-Da-Silva-Lopes V. Anomalias craniofaciais: descrição e avaliação das características gerais da atenção no Sistema Único de Saúde. Cad Saude Publica 2006; 22(5):913-922.

18. Monlleó IL, Mossey PA, Gil-da-Silva-Lopes VL. Evaluation of craniofacial care outside the brazilian reference network for craniofacial treatment. Cleft $\mathrm{Pa}$ late Craniofac J 2009;46(2):204-211.

19. American Cleft Palate-Craniofacial Association. $\mathrm{Pa}$ rameters for evaluation and treatment of patients with cleft lip/palate or other craniofacial anomalies. Revised ed. Chapel Hill: American Cleft Palate-Craniofacial Association; 2009. p.16.

20. Queirós FC, Wehby GL, Vila-Nova C, Silva LPA. Standards of care for patients with orofacial clefts in Brazil: starting a necessary debate. J Cranio Max Dis 2013; 2(2):122-129.

21. Yazdy MM, Honein MA, Rasmussen SA, Frias JL. Priorities for Future Public Health Research in Orofacial Clefts. Cleft Palate Craniofac J 2007; 44 (4):351357.

22. Fontes MIB, Almeida LN, Júnior GOR, Vieira-Filho JI, Santos KM, Anjos FS, Andrade AKM, Porciuncula, CGG, Oliveira, MC, Pereira RM, Vieira TAP, ViguettiCampos NL, Gil-da-Silva-Lopes VL, Monlleó IL. Local Strategies to Address Health Needs of Individuals With Orofacial Clefts in Alagoas, Brazil. Cleft Palate Craniofac J 2013; 50(4):424-431.

23. Silva RN. Características epidemiológicas de crianças portadoras de fissuras labiopalatinas atendidas no Hospital Infantil Albert Sabin, Fortaleza-CE [dissertação]. Fortaleza: Faculdade de Medicina; 2010.

24. Chor D, Lima CRA. Aspectos epidemiológicos das desigualdades raciais em saúde no Brasil. Cad Saude Publica 2005; 21(5):1586-1594.

25. Cassel CH, Daniels J, Meyer RE. Timeliness of Primary Cleft Lip/Palate Surgery. Cleft Palate Craniofac J 2009; 46(6):588-597.

26. Travassos C, Oliveira EXG, Viacava F. Desigualdades geográficas e sociais no acesso aos serviços de saúde no Brasil: 1998 e 2003. Cien Saude Colet 2006; 11 (4):975-986

27. Araújo JD. Epidemiological Polarization in Brazil. Epidemiol Serv Saude 2012; 21(4): 533-538.
28. Albuquerque MV, Viana ALD, Lima LD, Ferreira MP Fusaro ER, Iozzi FL. Desigualdades regionais na saúde: mudanças observadas no Brasil de 2000 a 2016. Cien Saude Colet 2017; 22(4):1055-1064.

29. Magalhães R, Burlandy L, Senna MCM. Desigualdades sociais, saúde e bem-estar: oportunidades e problemas no horizonte de políticas públicas sociais. Cien Saude Colet 2007; 12(6):1415-1421.

30. Carlson LC, Hatcher KW, Tomberg L, Kabetu C, Ayala $\mathrm{R}$, Burg RV. Inequitable acess to timely cleft palate surgery in low and middle-income countries. World J Surg 2016; 40(5):1047-1052.

31. Silva SF. Organização de redes regionalizadas e integradas de atenção à saúde: desafios do Sistema Único de Saúde (Brasil). Cien Saude Colet 2011; 16(6):27532762.

32. Mossey PA, Little J, Munger RG, Dixxon M, Shaw WC. Cleft lip and palate. The Lancet 2009; 374(9703):17731785.

33. Cubitt JJ, Van Lierde KM, Swan MC, Phill D. Global variation in cleft palate repair: an analysis of 352,191 primary cleft repair in low to higher-middle-income countries. Cleft Palate Craniofac J 2014; 51(5):553556.

34. Rafael RAA, Ribeiro VS, Cavalcante MCV, Santos, AM, Simões VMF. Relacionamento probabilístico: recuperação de informações de óbitos infantis e natimortos em localidade no Maranhão, Brasil. Cad Saude Publica 2011; 27(7):1371-1379.

35. Mello Jorge MHP, Laurenti R, Gotlieb SLD. Avaliação dos sistemas de informação em saúde no Brasil. Cad Saude Colet 2010; 18(1):7-18.

Artigo apresentado em 27/12/2018

Aprovado em 13/10/2019

Versão final apresentada em 15/10/2019

Editores-chefes: Romeu Gomes, Antônio Augusto Moura da Silva 
